\title{
A LONGITUDINAL STUDY OF FACEBOOK PRIVACY POLICIES
}

\author{
Alan Peslak, Penn State University, arp14@psu.edu \\ Mauri Conforti, Geisinger Health Systems, maconforti1@geisinger.edu
}

\begin{abstract}
Facebook has come a long way since its founding by Mark Zuckerberg and others in 2004. From a Harvard website for students, to the world leader in social media, Facebook has changed and grown enormously. Currently, 2.32 billion people are active on Facebook every month. The privacy of information it collects has been under increasing scrutiny. The manuscript examines the volatile Facebook privacy policies from its beginnings in 2005 to present day. The polices were collected via the Internet Archive and represent a selected day from each year from 2005 through 2018. A comprehensive qualitative, linguistics and sentiment analysis on these policies was performed. This review finds significant similarities and differences in Facebook privacy policies over the years. Overall, Facebook privacy policies have become increasingly complex and legalistic, but also more positive in sentiment and more individually oriented. Implications and opportunities for further research are presented.
\end{abstract}

Keywords: Sentiment analysis, Facebook, Linguistic analysis, LIWC

\section{INTRODUCTION}

From its humble college beginnings in 2004 Facebook has grown to be the most popular social media site in the world. Facebook is perhaps the most popular form of world communication after verbal and telephone communications. It may also be the most popular form of written communications in the world. According to Zephoria (2019) in March of 2018, there were over 2.32 billion monthly active Facebook users. This is a 23 percent increase from the prior year. Five new profiles are created every second. Twenty percent of all page views in the United States transpires on Facebook. Photo uploads total 300 million per day. With such ubiquitous usage, many organizations, governments, and individuals have begun to become more vigilant in monitoring and controlling the massive data that Facebook collects from its users. Significant fines and penalties have been levied based on improper usage of this information. Because of its omnipresence and popularity, it is a fertile area for research. This manuscript analyzes one area of this massive communications vehicle, the privacy policies of Facebook over time.

This analysis reviews the privacy policies of Facebook since its inception until present day. By analyzing the policies, the authors intend to provide a longitudinal qualitative analysis of how Facebook privacy has evolved and changed over the years. The paper examines the policies via linguistic, sentiment and textual analysis and present a detailed report on where and how Facebook privacy has changed from 2005 to 2018. This work builds on prior work of the authors especially regarding linguistic and sentiment analysis.

Linguistic, textual, and sentiment analyses are the review of written or verbal communications to determine specific characteristics of a communication. These characteristics can determine specific insights or meanings within a document, message, or speech that goes beyond the simple words in the communication. As a result, they can provide deeper understanding of the communicator's intent, bias, or personality thus framing the communication in a specific context and further clarify the communicator's full message. (Peslak, 2017). The study reviews each of the Facebook Privacy Policy changes through linguistic and sentiment analyses. 


\section{Issues in Information Systems}

Volume 20, Issue 1, pp. 213-223, 2019

\section{LITERATURE REVIEW}

The authors have been studying privacy policy and linguistic and sentiment analysis for years and have uncovered much literature supporting this research. Some of the following literature review has been presented in these other papers (Peslak, 2017, Peslak, 2016).

Sentiment evaluation and linguistic analysis are commonplace techniques of studies in communication analysis. The utilization of linguistic analysis and especially the use of LIWC (Linguistic and Word Count) software program for research functions has been substantial. Back, Kufner, and Egloff (2011) analyzed 11th of September communications via LIWC. Cordova, Cunningham, Carlson, and Andrkowski (2001) used LIWC to research how individuals adjusted to having breast cancers. Robinson, Navea, and Ickes (2013) used LIWC analysis to successfully estimate course performance based on introductions written by students at the beginning of a semester.

LIWC software (Pennebaker, Booth, Boyd, and Francis, 2015) is described as such: "The way that the Linguistic Inquiry and Word Count program works is fairly simple. Basically, it reads a given text and counts the percentage of words that reflect different emotions, thinking styles, social concerns, and even parts of speech. Because LIWC was developed by researchers with interests in social, clinical, health, and cognitive psychology, the language categories were created to capture people's social and psychological states. ... The text analysis module then compares each word in the text against a user-defined dictionary. As described below, the dictionary identifies which words are associated with which psychologically-relevant categories." (Pennebaker Conglomerates, 2015).

Both Sentiment Analysis on Facebook posts and Linguistic analysis using LIWC have been used before in the literature. Kramer (2012) studied Facebook posts via LIWC to determine how status updates affect emotional content on subsequent friends' posts. Getty et al. (2011) analyzed deceased persons' Facebook profile posts with LIWC. Farnadi et al. (2013) reviewed Facebook posts via LIWC to determine specific personality traits of individuals. There has been limited study of sentiment analysis within Facebook. Ortigosa et al. (2014) studied Facebook posts to determine users' sentiment polarity with a proposal to use these results to apply to e-learning.

Overall, sentiment analysis has been an increasingly important qualitative analysis tool. Bing Liu (2012, p.7) defines: "Sentiment analysis, also called opinion mining, is the field of study that analyzes people's opinions, sentiments, evaluations, appraisals, attitudes,". Sentiment Analysis is the review of written or other forms of communication or qualitative data to determine a quantifiable and comparable measure of some form of feeling in the communication or data. Pak and Paroubek (2010) pioneered the study of Twitter feeds for sentiment analysis.

Pang and Lee (2008) approaches whether a particular communication is positive or negative. Eguchi and Lavrenko (2006) suggest one of the first and still most used method of sentiment analysis is keyword analysis.

Many studies have been performed on privacy policies of Internet sites. The authors previously studied the Privacy Policies in several manuscripts (Peslak, 2016). After a comprehensive Google Scholar search, no instances of sentiment analysis or opinion mining of privacy policies in the literature were found. With so much information and so much activity, analysis of the major search engines is a fertile area of research. Our review is to analyze privacy statements of Facebook privacy policies over time using content analysis.

\section{METHODOLOGY}

Our overall research objectives included an examination of the relevant qualitative measures of Facebook privacy policies, how have they changed over time, and the impact on Facebook users.

To study the Facebook privacy polices over time it was first necessary to obtain past and current Facebook privacy policies. Of course, the current privacy policy of Facebook is posted on their website, but past policies are not. Fortunately, the Internet Archive (https://archive.org/web/) also known as the Wayback Machine has archived 351 billion webpages and past privacy policies were retrieved from the Internet Archive. As a sample, one dated page from each year from 2005 to 2018 was selected and retrieved the archived privacy policy from Facebook from that date. 
Though there may have been years where the policy may have changed at other times within the year, a once a year selection provided a reasonable representation of the volatility of Facebook privacy.

Once the policies were extracted, it needed to be determined how to analyze these polices. Three areas of analysis were chosen based on past work (Peslak 2016, 2017) and other qualitative relevant literature.

There were three general areas studied: Overall Content, Specific Word and Key Word Content, and Sentiment and Linguistic Analysis. To analyze Overall content, several tools were utilized. The online tool Copyleaks was first used to determine overall content changes from year to year. Microsoft Word was used to determine Reading grade level, complexity and word count. For sentiment and linguistic analysis, two tools were used. LIWC was used to determine key variables over time including clout, analytic, tone, and authenticity. IBM Watson Sentiment Analysis was used for sentiment (positive/negative) evaluation to determine degree of positive and negative content. For keyword and other specific content Voyant Tools was utilized, as well as specific author reviews of each policy. Another tool used in the study was Microsoft Excel for charting and other analyses.

\section{FACEBOOK 2005 OVERVIEW}

Facebook's first privacy policy was published in June of 2005. At that time the site was known as Thefacebook. The word count was relatively low at 1,004 words. The policy introduction states that it was designed to assist in understanding how they collect and use personal information that is provided to them and to assist in making informed decisions when using Thefacebook website. The policy was fairly simple at that time.

Some of the relevant items included in the 2005 policy follow. Through registering your account, you provide Thefacebook with personal information and Website information including browser type and IP address. You create your own profile and privacy settings, then the information is displayed to people/groups you select. They may send new information they think you may like occasionally. If you don't want to receive any you may email them. They use information collected from other sources to supplement your profile unless you opt-out in your privacy setting. They do share information with third parties to help bring services Thefacebook offers. Subpoenas and court orders can require them to disclose information. If the ownership changed, all information would be transferred. The site may contain links to other sites and Thefacebook is not responsible for their privacy practices. Advertisements are delivered by advertising partners, who can download cookies to your computer to gain information on your advertisement history. Thefacebook does not control these cookies. Users may modify or remove any personal information by logging into their account. Precautions are taken to protect user's information on a secured server behind a firewall.

\section{RESULTS OF LONGITUDINAL ANALYSIS}

From this simple first policy, Facebook has made significant and frequent changes over the years. The very first graph shown in Figure 1 is an illustration of how much Facebook privacy policies have changed over the last 14 years. The dark and light illustrate the percentage of the document that has remained identical versus the percentage of how much has changed or is different. There are also some small percentages of minor changes and related meanings. Facebook has had a very volatile Privacy Policy over the years, with significant changes in 2006 and 2007 and dramatic changes in 2010, 2012, and 2015. A detailed description of these changes follows. The source of these data was from CopyLeaks online software (2019). 


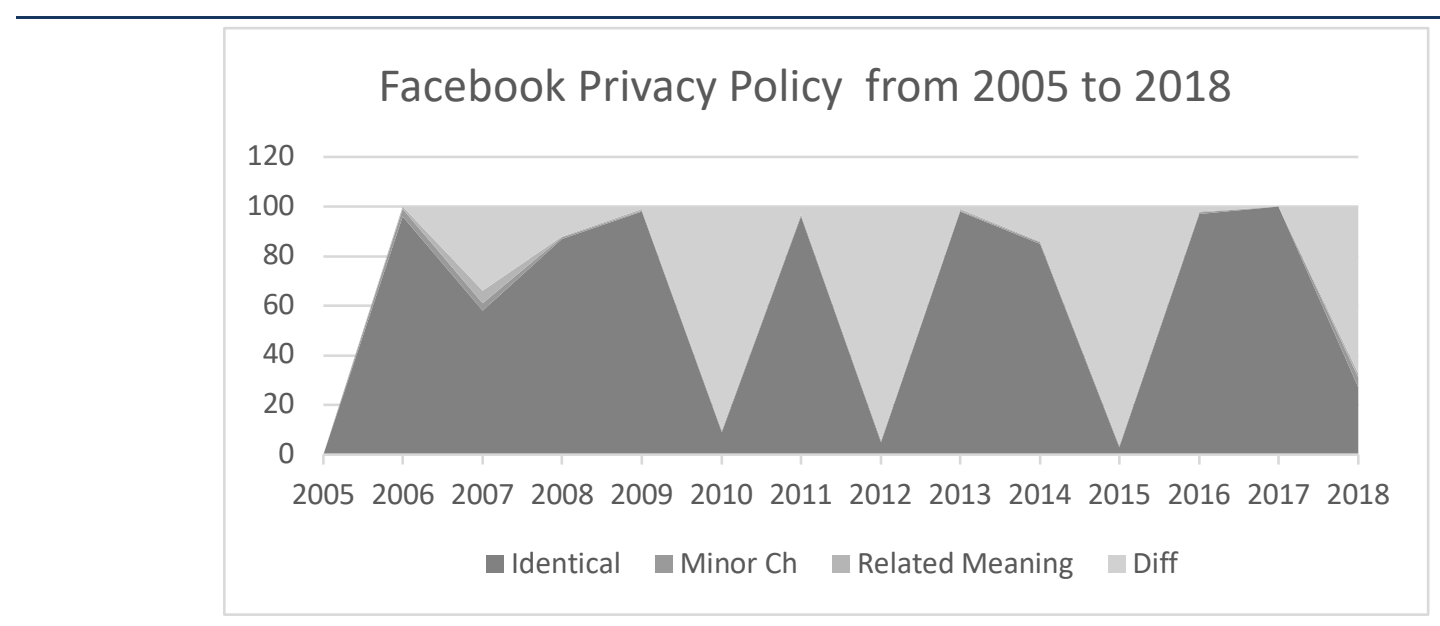

Figure 1. Facebook Privacy Changes

Table 1. Facebook Longitudinal

\begin{tabular}{|l|l|l|l|l|l|l|l|l|}
\hline Year & $\begin{array}{l}\text { Word } \\
\text { Count }\end{array}$ & Analytic & Clout & Authentic & Tone & Words/Sentence & $\begin{array}{l}\text { Grade } \\
\text { Level }\end{array}$ & Sentiment \\
\hline 2005 & 1004 & 72.68 & 99.00 & 42.85 & 78.65 & 20.42857 & 12.6 & 0.67 \\
\hline 2006 & 1167 & 75.60 & 99.00 & 48.25 & 75.13 & 21.18182 & 12.7 & 0.59 \\
\hline 2007 & 3099 & 72.80 & 99.00 & 36.99 & 75.47 & 23.52672 & 13.2 & 0.57 \\
\hline 2008 & 3774 & 73.18 & 99.00 & 39.28 & 78.65 & 25.47619 & 13.9 & 0.6 \\
\hline 2009 & 3786 & 73.71 & 99.00 & 39.05 & 78.90 & 25.43919 & 13.8 & 0.6 \\
\hline 2010 & 5900 & 59.20 & 99.00 & 26.53 & 72.71 & 19.7953 & 12.8 & 0.58 \\
\hline 2011 & 5977 & 59.26 & 99.00 & 26.78 & 73.01 & 19.78808 & 12.8 & 0.59 \\
\hline 2012 & 8896 & 56.69 & 99.00 & 28.56 & 66.01 & 24.52368 & 11.8 & 0.55 \\
\hline 2013 & 9522 & 56.26 & 99.00 & 30.73 & 67.27 & 25.08511 & 12 & 0.54 \\
\hline 2014 & 9430 & 55.51 & 99.00 & 30.20 & 67.65 & 25.87258 & 12.2 & 0.56 \\
\hline 2015 & 2716 & 53.76 & 99.00 & 25.49 & 85.17 & 26.36893 & 11.9 & 0.69 \\
\hline 2016 & 2712 & 52.63 & 99.00 & 26.84 & 84.36 & 26.3301 & 11.9 & 0.69 \\
\hline 2017 & 2712 & 52.63 & 99.00 & 26.84 & 84.36 & 26.3301 & 11.9 & 0.68 \\
\hline 2018 & 4245 & 55.76 & 99.00 & 26.96 & 72.52 & 28.3 & 12.9 & 0.73 \\
\hline Source & & & & & & & \\
\hline
\end{tabular}

Source: LIWC and Voyant Tools and Microsoft Word

Relevant analytical measures are all included in Table 1. One measure which reflects the complexity of a policy is word count (figure 2). The higher the word count the more a user must read through to fully understand what the policy represents. The first Facebook policy of 2005 was relatively short at 1,004 words. But their policy ballooned to nearly 9,000 words by 2012 . Recently, the level has decreased significantly but remains somewhat high at 4,245 words, which is a marked increase again over 2015 through 2017. It is unlikely users are all reading these policies to determine how their information is being used.

Some of the specific changes affecting word count follow:

In 2007, word count was 3,099 increasing from 1,004 words in 2005.

The Information We Collect section grew to eight paragraphs. Specific areas added include: 
1. Facebook collects information such as messages, queries, groups and events so you can return to prior messages and easily see your friend list.

2. Users posting contents is at your own risk. No security measures are perfect, Facebook cannot control the actions of others.

3. "Any improper collection or misuse of information provided is a violation of Facebook terms and agreements".

4. The Invitation service is used to tell a friend about the site, sending an email once inviting them to visit the site. The email will be stored to register a friend connection and track the success of the referral program.

In 2010, word count again rose to 5,900. The Information We Collect section expanded to four distinct sections: Information We Receive, Information Received from Third Parties, Sharing Information on Facebook, Information You Share with Third Parties.

In 2012, word count rose to its highest at 8,896 words. How information is collected and used increased again and is now included in seven separate sections: Information We Receive and How It Is Used, Information We Receive About You, Information Others Share, Other Information We Receive About You, Public Information, Information That Is Always Publicly Available, User Names and User IDs.

In 2015, word count significantly decreased to 2,716. The information sections collapsed to three sections: Information We Collect and How It Is Used, What Kinds of Information Do We Collect, How Do We Use This Information.

In 2018, word count rebounded to 4,245 as the information collected sections again increased and included: Things You and Others Do to Provide Information, What kind of Information We Collect, Information and Content You Provide, Networks and Connections You provide, Your Usage, Information About Transactions Made on Our Products, Device Information, Information from Partners, How Do We Use This Information.

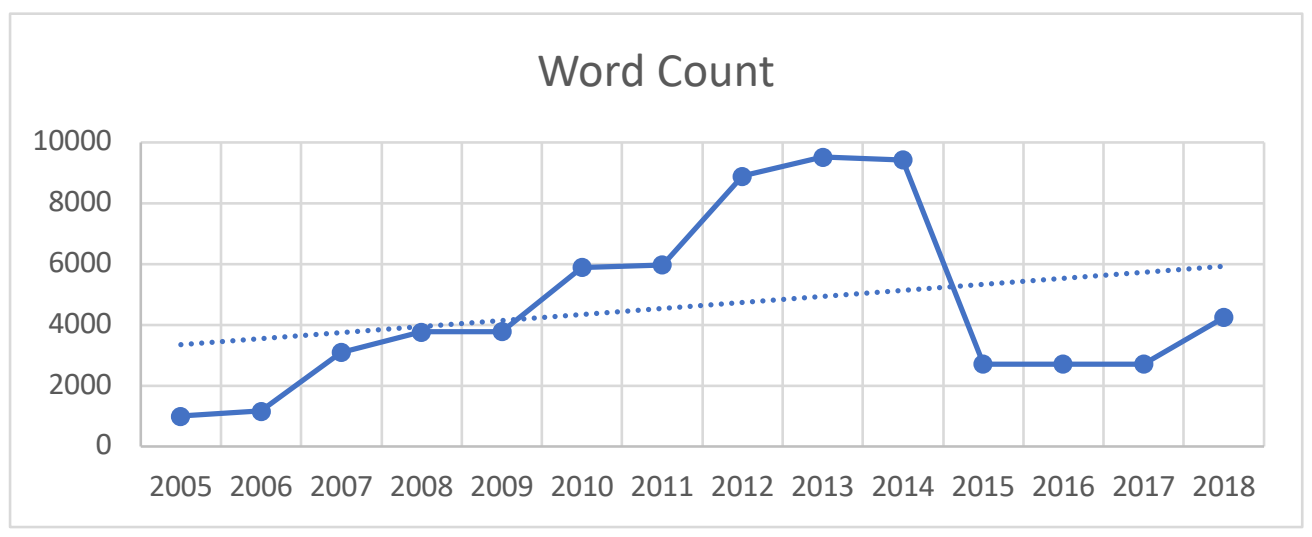

Figure 2. Facebook Word Count

In conjunction with the sheer word count, another measure of complexity is the reading grade level of the policies. As Figure 3 shows, Facebook has always had a high-grade level for its privacy policies. Though the measure is down slightly over the period, the expectation is still at a $12^{\text {th }}$ grade level or above. This is a very high level for general understanding by the majority of the population. For example, Hutchinson, Baird, and Garg (2016) suggest that Internet medical information should be written at a $6^{\text {th }}$ to $7^{\text {th }}$ grade level for general understanding. Clearly, the Facebook privacy policies far exceed this number. Another measure of complexity is words per sentence. Table 1 shows a steadily increasing number of words per sentence in the policies, indicating more textual complexity. 


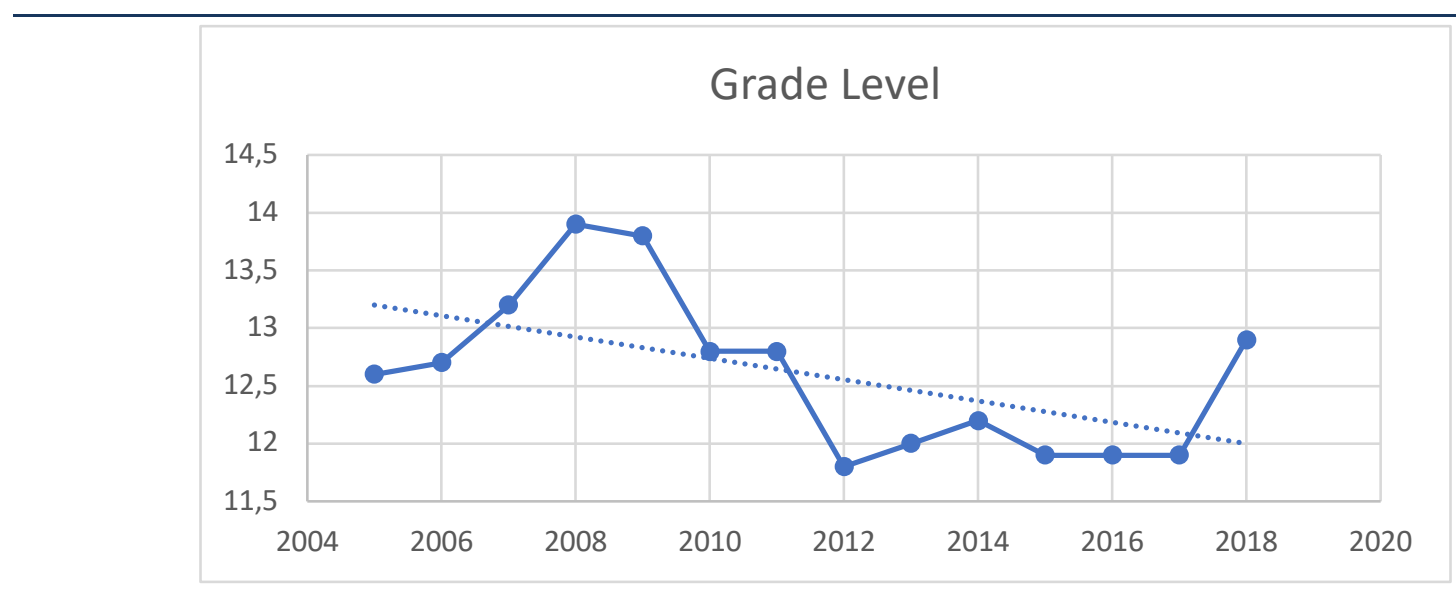

Figure 3. Facebook Reading Grade Level

As noted, the Facebook privacy policies posts were analyzed via IBM Watson sentiment analysis. Figure 4 represents the results of the analysis. This and all scores are presented on a 1-100 scale with 0 being lowest and 100 highest. For sentiment, a rating of zero would indicate very negative valence or sentiment, i.e. negative or bad feelings; 100 would represent very positive valence or sentiment, i.e. positive or good feelings. The table shows the sentiment score for each Facebook privacy policy. Overall, Facebook privacy policies have always shown positive sentiment and this positive tenor has been increasing over the years, as the positive slope on the trend line illustrates. Facebook has attempted to set an overall emotional impression of goodwill. The authors searched for prior research on sentiment analysis of corporations' privacy policy but did not find prior studies. The authors did review Google privacy policy for sentiment and found that overall Google privacy policy sentiment started much lower than Facebook at .40 and has remained lower throughout the relevant period. Google has shown an upward trend though, rising to .63 in 2018 still well below the sentiment of Facebook at .73.

Sentiment has been positive, friendlier, more enjoyable over the years. In 2015, specific positive passages were added:

1. "We are passionate about creating engaging and customized experiences for people". "Use all the information to help provide and support their services."

2. "We give you the power to share" is part of their mission.

3. Use of your information to communicate with you through marketing advertisers and vendors ads so the content is as relevant, enjoyable and interesting to the user. This year's policy approach seems to be that the experience is all about you.

4. In condensing the privacy policy, additional links were added to access legal, rights and security information. The visible content is more about people you share with, seeing content others share, apps, and third-party websites. Even though much of the information is in prior policies, the verbiage is more evident that using Facebook is all about you and personalized experiences. Many legalities and formalities are now in separate linked pages. 


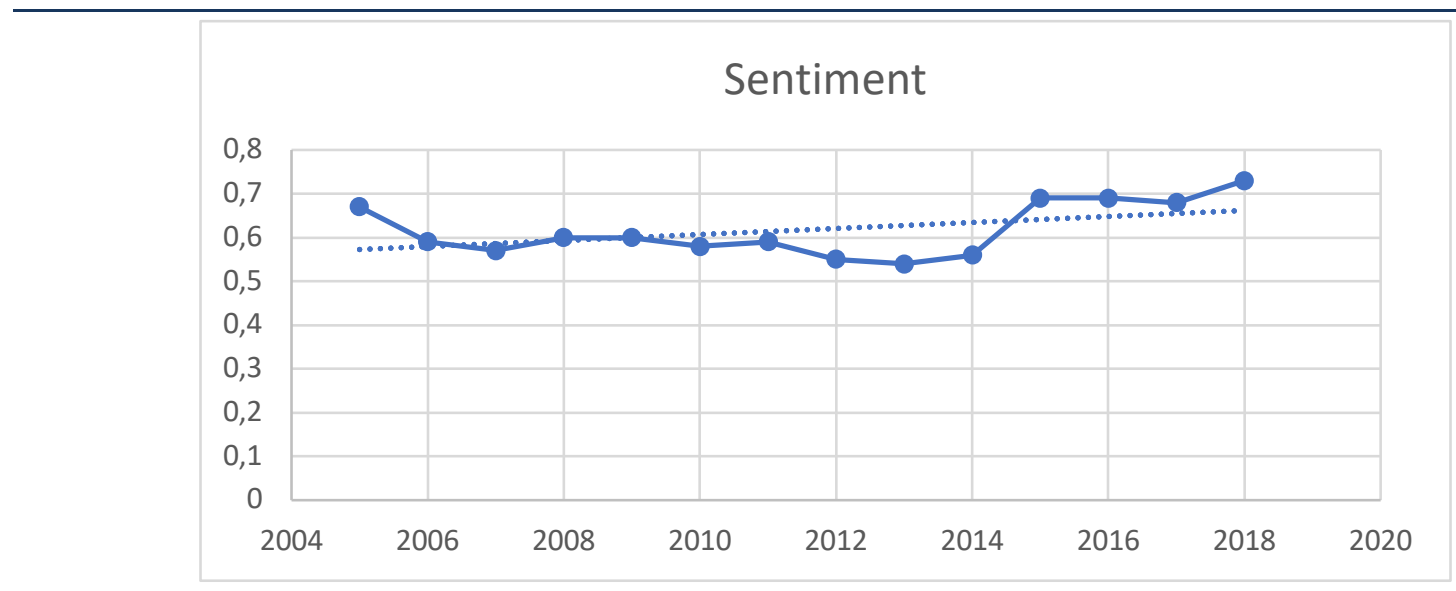

Figure 4. Facebook Sentiment

The rest of the analyses all use the results of LIWC. According to (Pennebaker, Booth, Boyd, and Francis, 2015) "Analytical thinking ------ a high number reflects formal, logical, and hierarchical thinking; lower numbers reflect more informal, personal, here --- and --- now, and narrative thinking.". The analytic score for Facebook privacy policies are high but this has been on the decline. There appears to be more of a personal touch to the policy and somewhat less formal. The NY Times is very analytical at 93 but natural speech is only 18. (Pennebaker, Booth, Boyd, \& Francis, 2015) The Facebook privacy policy is much more formal than speech but below and more informal than the New York Times.

Over the years, the LIWC Analytical rating decreased, shown in Figure 5, changing to a more informal, personal, and individual-centered document.

A major decrease was found from 2007 to 2010 where two sections were added: How We Use Your Information and How We Share Information:

1. "To Serve Personalized Advertising to You" by sharing your interests to advertisers.

2. "To Make Suggestions" by using the information you provided, making suggestions of people you might want to connect with.

3. "To Help Your Friends Find You" by allowing use of information in search results.

4. "To Provide You with Services" by using third parties to assist in bringing you the services Facebook offers.

5. "When You Invite A Friend to Join" the content may contain other information such as who else is friends with you.

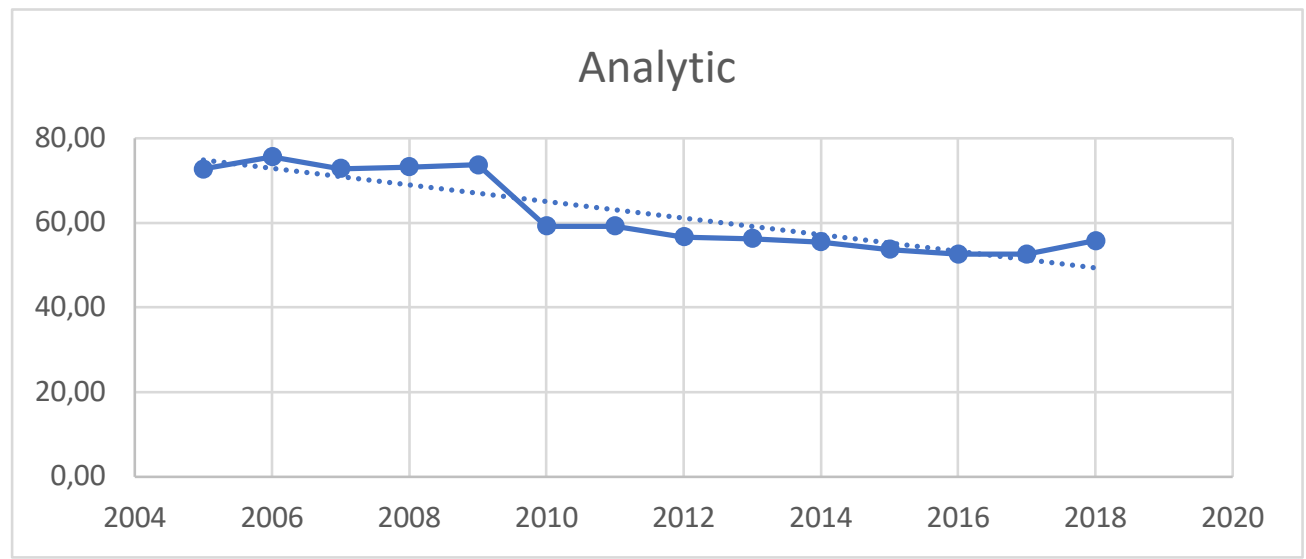

Figure 5. Facebook Analytical Rating 
Tone is the LIWC measure for sentiment and fairly well maps to the IBM Watson sentiment analysis. Facebook privacy policies have always been positive in tone and that tone level has had an increasing trend over the past 15 years as shown in Figure 6.

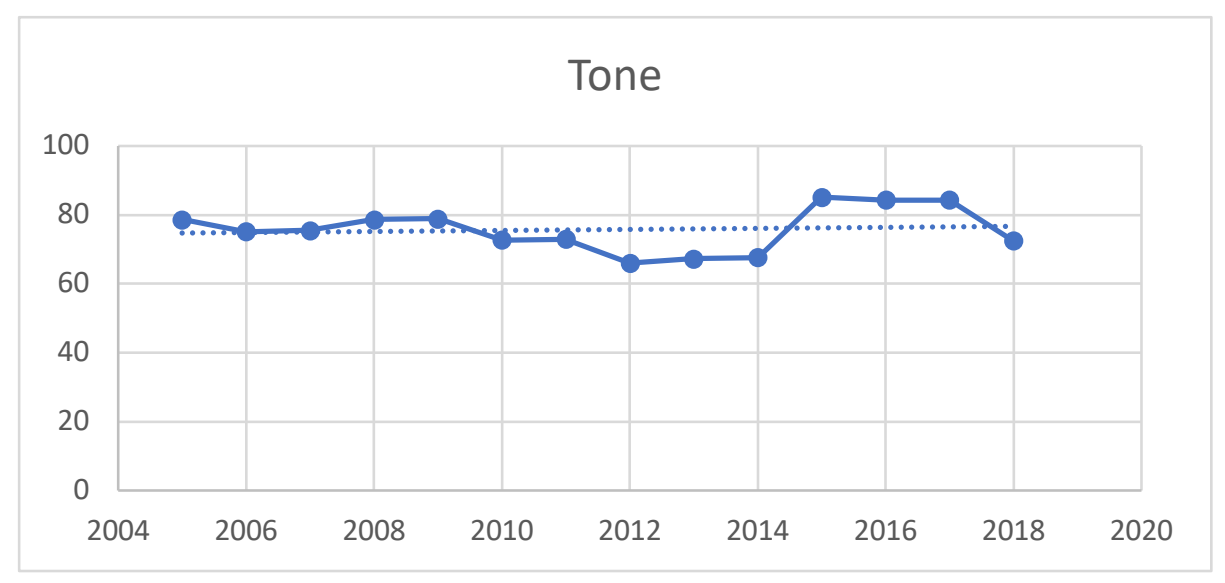

Figure 6. Facebook Tone

The authenticity (Authentic) has declined from 42.85 to 26.96 "Higher numbers are associated with a more honest, personal, and disclosing text; lower numbers suggest a more guarded, distanced form of discourse." (Pennebaker, Booth, Boyd, and Francis, 2015). Shown in Figure 7, Facebook privacy policy has taken a much more guarded tone over the years with a near steady decrease. The most precipitous drop was in 2010 when it went from 40 to below 30 . This would coincide with a more rigid and legal texture as Facebook has grown over the years and as the potential for legal issues would have increased. As a comparison, natural speech is very high at 61 and even Twitter is 50 . The Facebook policy is however in line with the New York Times which has an authenticity score of 25.

Authenticity decreased in 2010 from 2007 with a more guarded, legalistic, less personal approach.

1. "How You Can Change or Remove Information". In 2007, the section had one and half paragraphs. In 2010, the sections increased to six subtitles, new ones include: Deleting Uploaded Contacts, Backup Copies, NonUser Contact Information. The other sections expanded include: Deactivating or Deleting Your Account, Limitations on Removal.

2. "How We Protect Information", in 2007, the policy had one paragraph. In 2010, it was expanded to four subtitled areas.

- "Steps We Take to Keep Your Information Secure" Facebook does their best to keep information secure, but they need the users help. A secured server behind a Firewall, credit card numbers. and passwords are encrypted with SSL. Scanned for fraudulent behavior, abuse, inappropriate content, etc. For detailed information a user needs to visit the Security page link.

- They note there are "Risks Inherent in Sharing Information" therefore, "no security measures are perfect or impenetrable". Facebook is not responsible for any third-parties' actions in relation to these security measures.

- To "Report Violations" you are directed to a Help Page link

3. "Other Terms" section went from one paragraph to four expanded subtitles

- "Changes" section added links to Statement of Rights and Responsibilities and Facebook Site Governance Page.

- "Consent to Collection and Processing in the United States" section is a new section added this year.

- "Defined Terms" section is also new and includes meaning of terms used in Statement of Rights and Responsibilities link.

- "Helpful Links" section is new with 17 links listed for reference. In 2007, there are four sentences describing links with no actual links. 
Another legal section was added 2007 specifically addressing COPPA (Children's Online Privacy Protection Act of 2000). This was not included in the initial policy in 2005 and was dropped from the policy in 2012 and has not reappeared since.

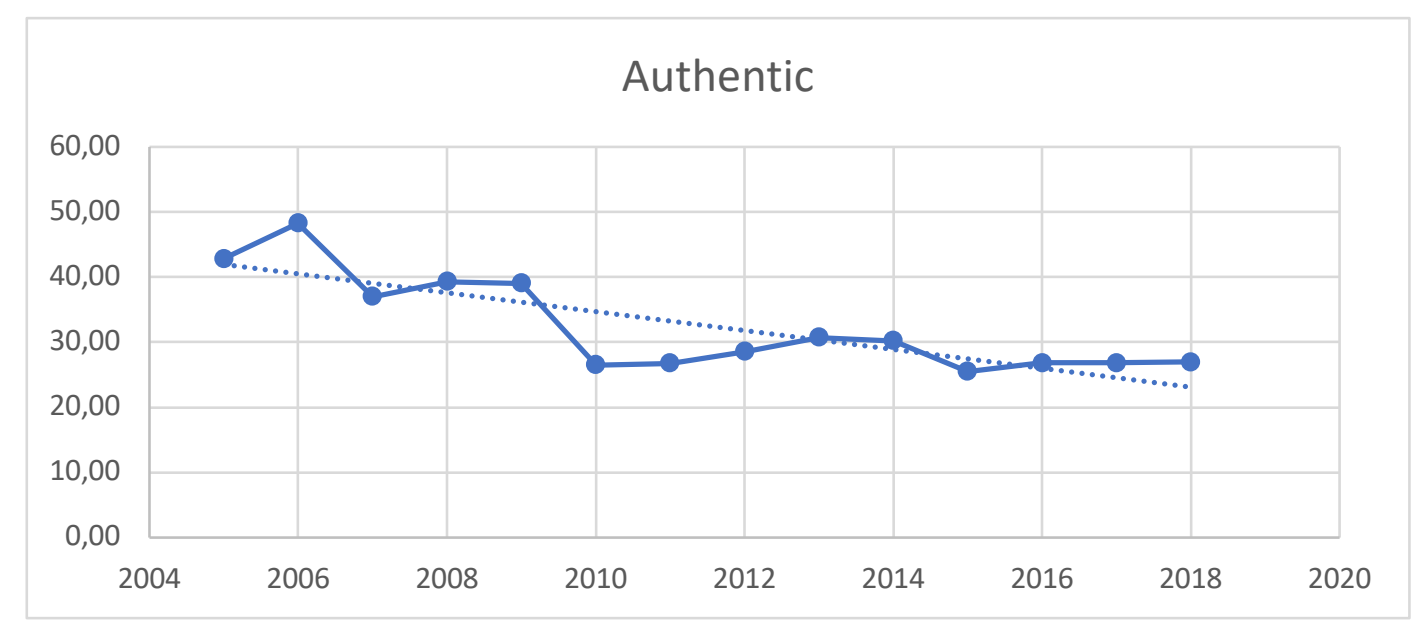

Figure 7. Facebook Authenticity

"Clout ------ a high number suggests that the author is speaking from the perspective of high expertise and is confident; low Clout numbers suggest a more tentative, humble, even anxious style." (Pennebaker, Booth, Boyd, and Francis, 2015). Clout is 99 and is same for all. Facebook privacy policies are confident and show high expertise. There is no tentative style in their documents at all. Since this is a policy, this is to be expected. As a comparison though, the New York Times only rates at 68 for Clout and Natural speech is only 52.

\section{SUMMARY AND CONCLUSION}

Overall, this manuscript can be viewed as a start to a qualitative analysis of Facebook privacy over the years since its inception. There were several significant takeaways from our study. The complexity of the Facebook privacy policy document based on reading grade level has decreased somewhat over its lifespan but remains high, currently at a year 13 grade level. This is a very high level for general understanding by the majority of the population.

Facebook privacy policies have experienced significant volatility over the 14 years of its existence with widely varying content and word count. It has been difficult to keep up with the ever-changing terms and many will have failed to read these constantly changing rules. Word count remains very high at over 4,000 words and includes links to other content. It is expected that few will read this detailed content. Those that read the policy will most likely scan through the document and few will access the numerous links. As a result of these complexities, most do not know what they are agreeing to in terms of privacy protection or lack thereof.

Overall Facebook sentiment is strongly positive and has risen over the lifespan. Facebook has attempted to set an overall emotional impression of goodwill. Facebook has become more informal and personal over the lifespan; the verbiage is more evident that using Facebook is all about you and personalized experiences. Many legalities and formalities are now in separate linked pages. This approach gives the impression that the experience is not focused so much on legalities and protection of the company, as the overall positive personal experience for the user. Most users will not access the legalities in the separate links.

But Authenticity has decreased over the lifespan and is now very guarded and legalistic. A more rigid and legal texture has emerged as Facebook has grown over the years and as the potential for legal issues would have increased. They also have more security measures in place to keep user information protected using Firewalls and password encrypting. 


\section{Issues in Information Systems}

Volume 20, Issue 1, pp. 213-223, 2019

Further research can be undertaken to further refine and understand how the policies have changed and how they further affect individual privacy. With a new overhaul of Facebook announced on May 1, 2019 as this manuscript was written, the authors look forward to also further expanding how this behemoth of a social communication platform will affect individual and personal privacy.

\section{REFERENCES}

Back, M. D., Küfner, A. C., \& Egloff, B. (2011). Automatic or the people? Anger on September 11, 2001, and lessons learned for the analysis of large digital data sets. Psychological Science, 22(6), 837-838.

CopyLeaks (2019) https://copyleaks.com/compare

Cordova, M. J., Cunningham, L. L., Carlson, C. R., \& Andrykowski, M. A. (2001). Social constraints, cognitive processing, and adjustment to breast cancer. Journal of consulting and clinical psychology, 69(4), 706.

Eguchi and V. Lavrenko, (2006). Sentiment retrieval using generative models, in Proceedings of the Conference on Empirical Methods in Natural Language Processing (EMNLP), 345-354.

Facebook (2005, 2006, 2007, 2008, 2009, 2010, 2011, 2012, 2013, 2014, 2015, 2016, 2017, 2018) Facebook Privacy Policy.

Farnadi, G., Zoghbi, S., Moens, M. F., \& De Cock, M. (2013, January). Recognising personality traits using facebook status updates. In Proceedings of the workshop on computational personality recognition (WCPR13) at the 7th international AAAI conference on weblogs and social media (ICWSM13). AAAI.

Getty, E., Cobb, J., Gabeler, M., Nelson, C., Weng, E., \& Hancock, J. (2011, May). I said your name in an empty room: grieving and continuing bonds on facebook. In Proceedings of the SIGCHI Conference on human factors in computing systems (pp. 997-1000). ACM.

Hutchinson, N., Baird, G. L., \& Garg, M. (2016). Examining the reading level of internet medical information for common internal medicine diagnoses. The American journal of medicine, 129(6), 637-639.

IBM (2019) IBM Watson Natural Language Understanding https://www.ibm.com/watson/services/natural-languageunderstanding/

Internet Archive https://archive.org/web/

Kramer, A. D. (2012, May). The spread of emotion via Facebook. In Proceedings of the SIGCHI Conference on Human Factors in Computing Systems, 767-770, ACM.

Liu, B. (2012). Sentiment analysis and opinion mining. Synthesis lectures on human language technologies, 5(1), 1167.

Microsoft Word Readability Statistics (2019) Microsoft Corp.

Ortigosa, A., Martín, J. M., \& Carro, R. M. (2014). Sentiment analysis in Facebook and its application to e-learning. Computers in Human Behavior, 31, 527-541.

Pak, A., \& Paroubek, P. (2010). Twitter as a corpus for sentiment analysis and opinion mining. In LREc 10, 13201326.

Pang, B., \& Lee, L. (2008). Opinion mining and sentiment analysis. Foundations and trends in information retrieval, 2(1-2), 1-135. 


\section{Issues in Information Systems}

Volume 20, Issue 1, pp. 213-223, 2019

Pennebaker Conglomerates (2015) LIWC How It Works. http://liwc.wpengine.com/how-it-works/

Pennebaker, J.W., Booth, R.J., Boyd, R.L., \& Francis, M.E. (2015). Linguistic Inquiry and Word Count: LIWC2015. Austin, TX: Pennebaker Conglomerates (www.LIWC.net).

Peslak, A. (2017) From Muslim To Mindfulness: Twitter Retweets Based on Sentiment And Linguistic Analysis Of Selected Current Keywords Issues in Information Systems, 18(2), 48-58.

Peslak, A. (2016). Do Social Networking Sites Privacy Policies Differ? A Linguistic Analysis Of The Ten Most Popular Social Networking Sites, Issues in Information Systems 17(7), 99-106.

Robinson, R. L., Navea, R., \& Ickes, W. (2013). Predicting final course performance from students' written selfintroductions: A LIWC analysis. Journal of Language and Social Psychology, $0261927 X 13476869$.

Sinclair, Stéfan and Geoffrey Rockwell, 2016. Voyant Tools. Web. http://voyant-tools.org/.

Voyant Tools (2019). http://www.voyant-tools.org

Zephoria (2019) The Top 20 Valuable Facebook Statistics - Updated March 2019 https://zephoria.com/top-15valuable-facebook-statistics/ 